\title{
What Happens When Children with Fetal Alcohol Spectrum Disorders Become Adults?
}

\author{
Eileen M. Moore $^{1} \cdot$ Edward P. Riley $^{1}$
}

Published online: 24 June 2015

(C) Springer International Publishing Switzerland 2015

\begin{abstract}
The range of structural abnormalities and functional deficits caused by prenatal alcohol exposure (PAE) are referred to as fetal alcohol spectrum disorders (FASDs). The disabilities associated with FASDs are said to be lifelong, but we know relatively little regarding outcomes beyond childhood and adolescence. Many of physical, brain, and neurobehavioral features that are present in children with FASDs will endure to adulthood. However, some features may diminish or change over time. Furthermore, secondary disabilities, such as school dropouts, trouble with the law, and substance/ alcohol abuse problems are common in young adults with FASDs. The health consequences associated with PAE in the human adult are unknown, but animal models suggest that they may be more susceptible to chronic diseases such as hypertension, diabetes, immune dysfunction, and cancer. More research is needed to understand the lasting effects of PAE on adults and the developmental trajectories of FASDs.
\end{abstract}

Keywords Fetal alcohol spectrum disorders · Adult · Health · Cognition · Behavior

\section{Introduction}

Prenatal alcohol exposure (PAE) can produce a spectrum of effects, including birth defects, craniofacial anomalies, growth

This article is part of the Topical Collection on Fetal Alcohol Syndrome

Eileen M. Moore

eimoore@mail.sdsu.edu

Edward P. Riley

eriley@mail.sdsu.edu

1 Center for Behavioral Teratology, San Diego State University, 6330 Alvarado Court, Suite 100, San Diego, CA 92120, USA retardation, and central nervous system dysfunction. Collectively, these outcomes are referred to as fetal alcohol spectrum disorders (FASDs). Alcohol's teratogenic effects are said to have a lifelong impact; however, little is known about FASDs beyond adolescence and young adulthood. A PubMed literature search for "fetal alcohol" restricted to humans revealed over 3000 references but the majority of this research examined children. In this review, we examine the literature on human adults with FASDs, including changes in physical and facial phenotypes, behavior and cognition, mental health and adaptive living outcomes, and neuroimaging findings. We outline the possible long-term health consequences of PAE based upon preclinical work. Hypotheses are offered on the trajectory of FASDs and possible mediating factors that may influence outcomes.

\section{Facial and Physical Phenotype}

A diagnosis of fetal alcohol syndrome (FAS) requires the presence of specific facial features, growth retardation, and evidence of central nervous system dysfunction [1-5]. However, Streissguth and colleagues [6] described 61 individuals with FAS spanning ages 12-40 years and found that the characteristic features of FAS were less distinctive after puberty. The deficiency in weight was not as substantial but the shortened stature persisted and many continued to display microcephaly.

Spohr et al. [7] conducted a 20-year follow-up on 37 individuals with PAE histories. These individuals were diagnosed early in life with either FAS or fetal alcohol effects (FAE), the term previously used to describe an individual affected by alcohol but not meeting the full criteria for FAS. The average age at first assessment was $3 \pm 2$ years and at follow-up $23 \pm$ 4 years. Many of the distinct facial features observed at the first assessment diminished over time; however, the thin upper 
lip persisted into young adulthood. Furthermore, while the majority of the sample was below the 3rd percentile for height, weight, and/or body mass index (BMI) at the initial assessment, many displayed "catch-up" growth for these measures. For example, $85 \%$ of this sample was below the 3 rd percentile for BMI initially but during young adulthood only $15 \%$ fell below this level. Head circumference was less prone to normalization across time; however, the percentage of individuals who fell below the 3rd percentile still dropped from $80 \%$ initially to $50 \%$ at follow-up.

Individuals are generally diagnosed with FASDs in childhood. Adults can be evaluated and diagnosed as well $[8,9]$, although there are challenges including obtaining reliable alcohol exposure histories and changes in the physical phenotype among others. More research is necessary to define the physical phenotype in adulthood but currently the research suggests that microcephaly, a thin upper lip, and shortened stature are persistent features. In an adult who has not previously been evaluated for FASDs, these features could alert a health care provider that such an evaluation should be considered. Behavior and cognition should also be assessed in an adult who is being evaluated for FASDs, but more knowledge about the neurobehavioral effects of PAE in adults is necessary to inform clinical practice.

\section{Behavior and Cognition}

Numerous reports on neurobehavioral deficits in children and adolescents with FASDs exist [10]. Work in adults is sparser, although a few studies have explored neurobehavioral outcomes during young adulthood. To date, behavioral problems, motor function, attention, learning and memory, executive function, theory of mind, and sense of coherence have been evaluated in adults with FASDs. Much more research is necessary to determine the specific cognitive and behavioral strengths and difficulties present in adults with PAE, especially for adults age 30 and above, as almost no data exists for this age group.

In a longitudinal study of the effects of PAE, a positive relationship was observed between alcohol exposure and behavioral problems at 22 years, as measured by the Adult Self Report [11]. Greater alcohol exposure was associated with increased total problems, as well as problems on internalizing, externalizing, attention, and critical items scales.

Motor functioning has been evaluated in two samples of young adults with PAE [12]. In a clinical sample of young adults (19-37 years) who were diagnosed with FAS $(n=30)$, FAE $(n=30)$ or nonexposed controls $(n=30)$, those with FASDs demonstrated poorer balance; were slower to perform finger, hand, and foot movements; and made more motor sequencing errors. However, in a prospective sample of young adults (24-27 years) with a range of PAE (from none to heavy), the dose response effect of alcohol on motor function was weak [12].

Visual and auditory attention was assessed using four different tasks in young adults (average age 21 years, IQ $>70$ ) with FASDs $(n=11)$ as compared to nonexposed controls $(n=$ 9) [13]. The alcohol-exposed group performed poorer on all tasks compared to controls. In visual-attention tasks, alcoholexposed subjects had difficulties with focusing and sustaining attention, while in the auditory-attention tasks, they performed poorly when required to focus, sustain, and shift attention.

In a large sample of young adults ( $23 \pm 2$ years), verbal and nonverbal selective reminding memory tests were administered to measure encoding, learning rate, and forgetting [14]. This study included 47 individuals with physical effects of PAE (dysmorphic), 74 alcohol-exposed individuals without physical effects (nondysmorphic), 59 nonexposed controls, and 54 nonexposed individuals who had received special education services. Both the dysmorphic and nondysmorphic alcohol-exposed groups had more difficulty performing efficiently on learning and memory tasks than did controls, with the most prominent effect in the dysmorphic group. The performance decrement in alcohol-exposed subjects was attributed to learning problems associated with difficulties in encoding information rather than difficulties caused by forgetting previously encoded material. There was little difference between the alcohol-exposed groups and the nonexposed group that had received special education services, indicating a nonspecific deficit.

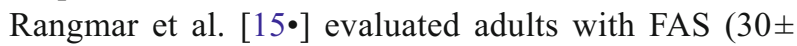
8 years) using several cognitive and social measures. Compared to controls, adults with FAS had shorter memory spans for Digit Span Forward and Backward and required more steps to solve the Tower of Hanoi, indicating they had poorer short-term memory, working memory, and planning ability. On Berg's Card-Sorting Test, a measure of cognitive flexibility/set-shifting sensitive to frontal lobe dysfunction, the FAS group had fewer total correct responses and made more non-perseverative errors, but did not differ from controls for perseverative errors [15•]. This conflicts with a prior study that showed increased perseverative errors in alcohol-exposed children [16]. This pattern of errors could indicate reduced efficiency or distractibility in adults with FAS but not an inability to alter responses following feedback.

After listening to stories about social situations, adults with FAS were less able to identify the suitability or ineptness of statements, despite adequately comprehending the stories on the Faux Pas Recognition Test [15•]. On Antonovsky's Sense of Coherence Scale, which measures how an individual feels about their environment and their place in it, adults with FAS had a lower Total Sense of Coherence score. The Total score is comprised of three subscales: (1) Manageability, measuring the degree to which an individual feels they have sufficient resources to cope; (2) Comprehensibility, whether an 
individual feels that the environment is structured and predictable; and (3) Meaningfulness, the sense that life is worthwhile. Adults with FAS did not differ from controls on the Comprehensibility or Meaningfulness subscales, but scored lower on Manageability [15•].

The research to date indicates that, compared to controls, adults with FASDs have increased behavioral problems; are perhaps less efficient and more distractible when completing tasks; have more difficulty with paying attention, learning, memory, planning, and analyzing social situations; and feel less confident that they have sufficient resources to cope with their environment. However, relatively few domains have been evaluated and these results are based on only a small number of measures. A more comprehensive assessment of neurobehavioral function in adults with PAE over a wider age range is necessary to inform clinical practice.

\section{Mental Health and Adaptive Living Outcomes}

The physical, cognitive, emotional, and/or social issues facing individuals with FASDs impacts their daily functioning. Secondary disabilities, conditions that a person is not born with but instead develops as a result of other problems, are common in FASDs. Streissguth et al. [17] noted that among adults with FASDs (21-51 years), $90 \%$ had mental health problems, $60 \%$ had trouble with the law, and $45 \%$ experienced drug and alcohol problems. In a clinical sample of 25 adults with FASDs (age 19-51 years; IQ 73-110) who were interviewed using the Structured Clinical Interview for DSM-IV Axis I Disorders (SCID) [18], $92 \%$ met criteria for an Axis-I disorder. The most common disorder was current or past alcohol or drug dependence, affecting $60 \%$. Forty-four percent met criteria for past or present depression, $40 \%$ reported psychotic symptoms, and anxiety or bipolar disorder was each observed in $20 \%$ [18]. Twenty-one of these subjects were also interviewed with the Structured Clinical Interview for DSMIII-R Personality Disorders (SCID-II) and almost half met criteria for at least one personality disorder [18]. The SCID and SCID-II were also administered to a larger $(N=400)$ prospective/nonclinical sample of young adults age 2427 years [19]. Exposure to one or more binge-alcohol exposures prenatally was associated with a greater than 2-fold risk for somatoform disorder; substance dependence or abuse; and paranoid, passive-aggressive, and antisocial personality disorders [19].

The cognitive deficits, behavioral problems, psychopathology, and other secondary disabilities associated with FASDs can impact everyday functioning or adaptive behavior and hinder achievement of adult independence. In their 20-year follow-up, Spohr et al. [7] found that only $13 \%$ of their sample of young adults with FASDs had ever held an "ordinary" job, despite $69 \%$ having had at least preparatory job training. Two thirds were in a dependent living or institutional setting and only $16 \%$ lived with a partner or had their own family. Similarly, in a report of 90 clinically referred adults with FASDs and an average age of 25 years, $70 \%$ were unemployed and dependent, and $80 \%$ needed assistance with daily living activities [20]. A functional assessment survey of the caregivers of adults with FASDs (age 17-43 years, IQ 45$120)$ found that a large number required a moderate $(37 \%)$ to high (44\%) level of care [21]. Eighty-one percent of adults with FASDs required greater than minimal levels of care although only $34 \%$ had an IQ score in the intellectually disabled range.

To determine if adults with FASDs (age 18-46 years, IQ 59-108) had low adaptive skills, their scores on the Adaptive Behavior Assessment System-II (ABAS-II) were compared to an age- and IQ-matched nonexposed group [22]. Adults with FASDs were found to have a significantly lower General Adaptive Composite score, calculated from the assessment of Conceptual, Social, and Practical Skills domains. The adults with FASDs were not significantly different from their age- and IQ-matched counterparts in their Social and Practical Skills; however, they had significantly lower Conceptual Skills scores. The Conceptual Skills domain probes selfdirection (e.g., planning for future), functional academics (e.g., managing time and money), and communication (e.g., expressing complex ideas).

Using the Total Population Register in Sweden, Rangmar et al. [23•] evaluated the psychosocial outcomes of 79 adults with FAS (18-47 years) as compared to the general population $(n=3160)$. The FAS group was more likely to have attended special education ( 25 vs. $2 \%$ ) and less likely to have completed postsecondary education ( 5 vs. $45 \%$ ). They were less likely to be employed ( 49 vs. $89 \%$ ) and more likely to be collecting a disability pension or using social welfare services ( 30 vs. $3 \%$ ). Consistent with previous reports, there were high rates of secondary disabilities in the FAS group and they were more likely to have received hospital care for alcohol or illicit drug use (13 vs. $3 \%$ ) or a psychiatric disorder (33 vs. $5 \%$ ). However, $81 \%$ of the adults with FAS had been placed in state care during their youth (vs. $4 \%$ in the general population). To account for this difference in background, the FAS group was compared to a subgroup of matched adults who were placed in state care prior to age $18(n=122)$. While the FAS group was more likely to have attended special education than the state care group ( 25 vs. $3 \%$ ), there were no differences between the groups in terms of completed education, income, self-support, or hospital care. Compared to the state care group, the FAS group was actually less likely to have a record or court conviction ( 28 vs. $55 \%$ ) or to have been convicted of a severe crime (6 vs. $30 \%$ ) [23•].

Adults with FASDs have a high rate of psychiatric and personality disorders, problems with drugs and alcohol, and difficulties with the law. They are also less likely to obtain a degree, have stable employment, and live independently. 
Importantly, Streissguth and colleagues [17] found that having a diagnosis before age 6 , a stable and nurturing home environment, and access to services may improve some of these outcomes. Research on how these secondary disabilities can be mitigated once an individual reaches adulthood is essential in order to improve the quality of life for adults with FASDs.

\section{Neuroimaging Findings}

Magnetic resonance imaging (MRI) studies examining children and adolescents with heavy PAE frequently report total brain volume reductions [24]. Chen et al. [25] investigated the impact of PAE on brain volume in young, prospectively identified adults (average age 23 years). Total brain size was reduced and the level of exposure negatively predicted brain size, which in turn positively predicted IQ score. Additionally, a number of subcortical and cortical regional volumes were negatively correlated with the extent of PAE, including the bilateral cerebellum, the posterior portion of the corpus callosum, the bilateral lingual gyri, and several portions of the frontal and parietal cortices. Most of these regions have also been implicated as specifically affected in children with FASDs, suggesting that these brain reductions persist into young adulthood.

Studies in children and adolescents have identified white matter as being sensitive to the effects of alcohol $[24,26]$. Diffusion tensor imaging (DTI) is a method used to indirectly study white matter microstructural integrity by measuring water diffusion. DTI measures include fractional anisotropy (FA), or how restricted the water diffusion is, and mean diffusivity (MD), an index of total diffusion. Generally, lower FA and higher MD are indicative of poorer white matter microstructural integrity. Ma et al. [27] examined the corpus callosum in dysmorphic alcohol-exposed young adults (1825 years) and found reduced FA and increased MD in the genu and splenium. Li et al. [28] examined the microstructure of the corpus callosum in both dysmorphic and nondysmorphic alcohol-exposed young adults (19-27 years) and found reduced FA and increased MD in the isthmus of the dysmorphic individuals.

Using functional MRI (fMRI), brain activity during an arithmetic task was examined in dysmorphic and nondysmorphic alcohol-exposed individuals (20-26 years) and compared to nonexposed controls [29]. Total accuracy on the arithmetic task was reduced in alcohol-exposed young adults. However, even after excluding subjects with $<50 \%$ accuracy, the alcohol-exposed dysmorphic individuals had reduced activation compared to controls in regions important for arithmetic processing: the left superior and right inferior parietal regions, and right medial frontal gyrus. The nondysmorphic group displayed intermediate activation that was not significantly different from either the control or the dysmorphic groups. Differences in activation in these regions may reflect a diminished ability for the alcohol-exposed subjects to recruit the appropriate network when performing the arithmetic task [29].

Several brain regions display greater activation at rest than during cognitive demand, and while engaged in cognitive tasks, these regions will actually show task-related deactivation. These regions have been deemed the Default Mode Network (DMN) and include the medial prefrontal cortex, posterior cingulate cortex, precuneus, inferior parietal lobules, and medial temporal regions. In the arithmetic task described above, dysmorphic alcohol-exposed young adults (1824 years) displayed attenuated task-dependent deactivation of the DMN as compared to controls [30]. Further, the timeseries correlation (functional connectivity) of the DMN was examined at rest, during which time subjects were instructed only to look at a fixation cross. Both alcohol-exposed groups displayed reduced connectivity at rest between the medial prefrontal cortex and the posterior cingulate cortex [30]. Task-related deactivation of the DMN may be related to attentional modulation [31] and a disconnection of the DMN could contribute to an attenuated task-related positive activation, lower task-related attentional modulation, and perhaps poorer task performance [30].

Only a few studies have used MRI to examine the effects of PAE on adult brain structure and function but it seems that at least some of the effects observed in childhood and adolescence persist into adulthood. Total brain size reductions, an impact on the microstructural integrity of the corpus callosum, and evidence of less efficient network activity during an arithmetic task and at rest have been reported in alcohol-exposed adults. Nothing is known about the development of white matter development beyond the effects observed in the corpus callosum, and again the age of the subjects is somewhat restricted. Many areas of cognitive functioning that are known to be difficult for children with FASDs, such as memory and executive function, have not yet been explored with functional neuroimaging techniques. Furthermore, research on how to promote healthy brain growth and enhance neural efficiency in individuals with PAE is lacking.

\section{Health Effects}

There are a few reports of poorer general health in alcoholexposed children, including lower immune function [32, 33], glucose intolerance, and hyperinsulemia [34]. A review of case studies suggests there may be a higher rate of some childhood cancers in FASDs than in the general population [35]. There is insufficient research on the health consequences in individuals with FASDs and nothing on adults. However, prenatal experiences can have a lasting impact on health via fetal programming [36]. Animal models support the notion that individuals with FASDs may be at a higher risk for some medical conditions. 
Adult mice (14-16 weeks) with prenatal/lactational alcohol exposure and controls were infected with the influenza virus [37]. The alcohol-exposed mice demonstrated increased weight loss, reduced survival rate, increased illness severity (elevated viral titers), and reduced T- and B-cell responses post-infection. This suggests that PAE has a long-lasting impact on virus susceptibility and immune response. Further exposure to alcohol during adulthood augmented some of these effects [37], which is concerning given the high rates of drug and alcohol problems in adults with FASDs (45 \%) [17].

PAE may also impact susceptibility for autoimmune diseases. Weinberg and colleagues [38] used an adjuvantinduced rheumatoid arthritis model in adult female rats with PAE. Injection of adjuvant, an immunostimulator, induces an inflammation of the joints. Following adjuvant administration, the alcohol-exposed rats did not significantly differ from controls in terms of the incidence of arthritis during the induction phase. However, during resolution, alcoholexposed females had an increased incidence of arthritis, greater severity, and a more prolonged course of inflammation compared to controls. Additionally, during arthritis induction the alcohol-exposed females had a blunted lymphocyte proliferative response to a polyclonal stimulant (Con A) and elevated basal adrenocorticotropic hormone levels compared to controls. This suggests that alcohol exposure alters both immune and neuroendocrine function, indicating both direct and indirect effects on chronic inflammatory processes [38].

Glucose homeostasis may also be impaired in adults with PAE, which may influence risk for diabetes. Pregnant rats were administered water or alcohol throughout pregnancy [39]. At 13 weeks, plasma glucose was measured in the nonfasting state and following a fasting glucose tolerance test. In alcohol-exposed rats, glucose levels were no different than controls during the nonfasting state, although insulin levels were increased. Following the glucose challenge, alcoholexposed rodents displayed both higher glucose and insulin levels. PAE may increase insulin resistance and glucose intolerance, both of which are factors in the pathophysiology of type 2 diabetes [39].

Gestational exposure to alcohol may produce hypertriglyceridemia. Rats exposed to prenatal alcohol via maternal liquid diet had elevated serum triglyceride levels during adulthood [40]. As male offspring aged, the alcohol-exposed rodents displayed increasingly elevated fasting triglyceride levels compared to controls. By 360 days of age, the average triglyceride levels of the alcohol-exposed animals were 3 -fold greater than that of pair-fed controls. No differences were observed in fasting triglyceride levels for female alcohol-exposed rats, excepting the females born to dams that experienced stress during pregnancy. The combination of prenatal alcohol and stress resulted in increased fasting triglycerides in both male and female alcohol-exposed rats [40]. Elevated triglycerides increase risk for cardiovascular disease, acute pancreatitis, and coronary heart disease [41].

PAE may increase susceptibility for some cancers. Polanco et al. [42] fed pregnant rats a liquid ethanol diet, a pair-fed liquid control diet, or an ad libitum diet. At 50 days of age, female offspring were administered the carcinogen $N$-nitroso$\mathrm{N}$-methylurea (NMU) and monitored for mammary tumor development. Sixteen-weeks post-NMU, alcohol-exposed rats had an increased number of mammary tumors compared to controls. By the study conclusion (23 weeks post-NMU), alcohol-exposed and pair-fed controls did not differ in the number of tumors, but alcohol-exposed rats had a higher number of tumors in comparison to ad libitum controls. While an increase in the number of carcinogen-induced tumors may not be a specific effect of PAE (as it is also seen in pair-fed animals), alcohol exposure does appear to speed the progression of mammary tumor development.

Murugan et al. [43] examined susceptibility to prostate tumorigenesis in male rats with PAE. Alcohol exposure was similar to that described above [42]; however, NMU and androgen treatment was used to induce prostate cancer [43]. Prostates were evaluated at 6 months of age to determine if the hormone and carcinogen treatment induced prostatic intraepithelial neoplasia (PIN), an abnormality that precedes a common form of prostate cancer. Control rats exhibited lowgrade PIN in the ventral prostate lobe and no change in histological composition of the dorsal lobe. By contrast, the same treatment induced high-grade PIN in the ventral prostate and low-grade PIN in the dorsal lobe of alcohol-exposed rodents. Together, these studies suggest that PAE can produce cellular changes that may make an individual more susceptible to carcinogenesis in adulthood.

Given the animal work, we might expect adults with FASDs to be more susceptible to common maladies such as influenza and have increased risk of several health conditions including autoimmune diseases, diabetes, cardiovascular diseases, and cancer. To our knowledge, no studies have examined the health consequences of PAE in human adults, an important topic sorely in need of research.

\section{FASDs Across the Lifespan}

Although some studies have described cognitive, behavioral, and brain consequences of PAE that are present in adulthood, the average age of subjects in those studies was around 25 years. However, brain maturation is a protracted process. Thus, while alcohol impacts brain development prenatally, the repercussions can result in a delay and/or deviation in brain development thereafter. In typical development during childhood, the brain has an overabundance of synaptic connections. Over time, these connections are reduced and refined via synaptic pruning and myelination. The trajectory of white matter development, such as myelination, is especially 
protracted [44]. DTI studies of white matter development show peak FA occurring in some tracts during the late thirties or early forties [45].

The timing of neural maturation processes is purposeful and coincides with sensory, cognitive, and behavioral development. Efficient neural networks and their associated functional processes mature in a regionally heterochronous manner. Prefrontal networks and higher order cognitive abilities, such as executive functioning, continue to develop as an individual ages into young adulthood and beyond. Working memory, cognitive control, and reasoning capacity, all components of executive functioning, increase as an individual ages from childhood into adulthood. Adult-level abilities to meet challenging executive function tasks are associated with more efficient transmission of information across widely distributed brain networks, which requires fast and synchronous connections across long distances [44]. Mature cognitive functioning requires a complex and coordinated development of gray and white matter to achieve efficient information processing.

Several studies have found that PAE can cause atypical brain maturation. In a longitudinal examination of cerebral cortex volume changes, PAE individuals displayed an atypically linear pattern of gray matter volume reduction across childhood and adolescence [46]. In a DTI study of longitudinal changes in white matter, alcohol-exposed children displayed a steeper decline in MD over time than their nonexposed peers in several fiber tracts [47]. With maturation, both controls and alcohol-exposed children displayed increases in white matter volume [48]. However, increased white matter volume predicted executive functioning improvement in alcohol-exposed subjects but not controls [48], which may suggest that for alcohol-exposed individuals' white matter development is particularly important for establishing efficient executive functioning networks. Following PAE, the brain's developmental trajectory and its relationship to cognition are different than what is seen in typical development.

Other pediatric psychiatric disorders show a developmental delay of brain maturation, including attention-deficit/hyperactivity disorder (ADHD). Cross-sectional neuroimaging studies of children with ADHD show reduced volume of frontal and parietal cortex, basal ganglia, corpus callosum, and cerebellum [49]. These same brain regions are reduced in children with FASDs [24]. Longitudinal studies of children with ADHD indicate that cortical regions, particularly in the frontal cortex, are delayed in achieving peak thickness [50]. Approximately one third of children with ADHD continue to be symptomatic in adulthood [51]. Those children who matured out of ADHD symptoms may have had delayed brain maturation that eventually reconciled. Clinical improvement of ADHD symptoms is associated with convergence of brain growth trajectories towards more typical development, whereas more progressive deviation from typical development is associated with persistence of ADHD symptoms [49].
If alcohol-exposed children have a delayed or slower rate of brain maturation, then it is possible that given time certain individuals with FASDs may eventually reconcile some deficits, as is seen in some cases of ADHD. Early identification and diagnosis, access to services, and having a stable/ nurturing home environment were protective against adverse outcomes [17]. Such positive environmental factors may facilitate brain growth and increase odds of developmental convergence. Adverse life experiences (e.g., physical or sexual abuse) and a lack of access to appropriate services predicted unfavorable outcomes [17]. Adverse childhood experiences impact the brain, cognition, and behavior [52] and could be associated with a divergent brain growth trajectory in children with FASDs.

Another possibility is that adults with PAE may undergo premature or more accelerated aging than nonexposed individuals. Evidence of atypical aging has been observed in genetic disorders associated with facial dysmorphia and cognitive impairment, such as Prader-Willi syndrome, Williams syndrome [53], and Down syndrome [54]. Additionally, adult individuals who survived childhood cancer [55], experienced childhood maltreatment [56], and those with prior traumatic brain injury [57] and numerous other insults across the lifespan also show evidence of atypical aging or accelerated cognitive decline. These insults, whether genetic or environmental, could also be thought of as diminishing an individual's physiologic, brain, and/or cognitive reserve capacity. Lower reserve capacity may interact negatively with the normal aging process resulting in atypical trajectories of functioning $[58,59]$. This may have implications for adults with PAE; however, the aging process in these individuals remains unknown and studies are needed to clarify outcomes.

There is accumulating evidence that the developmental trajectory is atypical in children with FASDs; however, it is unclear how this may affect the adult with PAE. A developmental delay or slower brain maturation rate for individuals with PAE warrants the possibility that some of their difficulties may reconcile over time. However, it is also possible that an individual with PAE will continue to display atypical trajectories across their lifespan. There is also the potential that adults with FASDs may undergo premature or accelerated aging. At present, we can only speculate about possible outcomes. Research on the effects of PAE across the lifespan, including middle and late adulthood, is necessary to determine the long-term needs of individuals with FASDs.

\section{Conclusion}

We know very little about the impact of PAE on the human adult. At least some of the neurobehavioral effects of PAE endure through young adulthood; however, some cognitive deficits that were present in childhood (e.g., perseverative 
errors; [16]) may not persist into adulthood [15•], indicating ongoing neuromaturation may result in at least some mitigation of deficits. Young adults with PAE have increased risks for mental health problems and secondary disabilities [17], which impact their ability to live independently [21]. However, beyond age 30 , nearly nothing is known about cognition and behavior and the health consequences are undetermined. There are a few studies that have examined the health of children and or adolescents but the majority of what is known about adults has come from work in animal models [37-40, 42, 43], which suggests that as individuals with FASDs age, they may be more prone to certain diseases (e.g., autoimmune, diabetes, hypertension, and cancer). More research is needed to understand the developmental trajectory of FASDs as well as the neurobehavioral sequelae and health risks associated with FASDs in adults.

While many adults with FASDs continue to struggle with lifelong disabilities, there are also numerous examples of adults with FASDs leading productive lives and utilizing coping strategies. The variability in outcomes highlights the need for more research in adults with FASDs so that we may better predict outcome and identify the high-risk individuals who are most in need of intervention. One distinct possibility is that alcohol-exposed individuals have atypical or delayed brain maturation. While typically developing individuals may reach adult-level cognitive abilities around age 25 years, alcoholexposed individuals may plateau later. Similar to those with ADHD, the brain may take longer to reach more adult levels of maturity. Executive function networks are among the last to mature, and for alcohol-exposed individuals, executive function improvement over time is associated with increases in white matter volume [48]. Given the protracted period of white matter development in typical development, perhaps alcohol-exposed individuals do not achieve their most efficient adult-level state for higher order cognitive networks until a later age, such as age 30 or beyond. While deficits related to structural anomalies such as smaller callosal or caudate volumes may endure, deficits related to deficient connectivity may improve over time. Another possibility is that adults with FASDs may experience atypical aging, similar to individuals with some genetic disorders or other early insults. To date, the majority of studies that examined adults with FASDs have assessed individuals less than 30 years of age, leaving us with mere conjecture at this point. With continued research into brain and cognitive development using an older sample of adults with FASDs, we will be able to test these hypotheses and eventually answer the question: what happens to children with FASDs when they become adults?

Acknowledgments Financial support was received from the National Institute on Alcohol Abuse and Alcoholism (NIAAA) grants U24 AA014811 to EPR and a K99 022661 to EMM, as well as an American Fellowship from the American Association of University Women
(AAUW) to EMM. We thank Leila Glass, M.S. for her helpful critique of this review and also the individuals with FASDs and their families who provided us the impetus for looking at the effects of prenatal alcohol on health and behavior in adults.

\section{Compliance with Ethical Guidelines}

Conflict of Interest Eileen M. Moore and Edward P. Riley declare that they have no conflict of interest.

Human and Animal Rights and Informed Consent This article does not contain any studies with human or animal subjects performed by any of the authors.

\section{References}

Papers of particular interest, published recently, have been highlighted as:

- Of importance

1. Chudley AE, Conry J, Cook JL, Loock C, Rosales T, LeBlanc N. Fetal alcohol spectrum disorder: Canadian guidelines for diagnosis. CanMed Assoc J. 2005;172(5):S1-S21.

2. Bertrand J, Floyd LL, Weber MK. Guidelines for identifying and referring persons with fetal alcohol syndrome. Morbid Mortal Wkly Rep Recomm Rep / Cent Dis Ctrl. 2005;54(11):1-14.

3. Astley SJ, Clarren SK. Diagnosing the full spectrum of fetal alcohol-exposed individuals: introducing the 4-digit diagnostic code. Alcohol Alcohol. 2000;35(4):400-10.

4. Astley SJ. Validation of the fetal alcohol spectrum disorder (FASD) 4-Digit Diagnostic Code. J Popul Ther Clin Pharmacol. 2013;20(3): e416-67.

5. Hoyme HE, May PA, Kalberg WO, Kodituwakku P, Gossage JP, Trujillo PM, et al. A practical clinical approach to diagnosis of fetal alcohol spectrum disorders: clarification of the 1996 Institute of Medicine criteria. Pediatrics. 2005;115(1):39-47.

6. Streissguth AP, Aase JM, Clarren SK, Randels SP, LaDue RA, Smith DF. Fetal alcohol syndrome in adolescents and adults. JAMA. 1991;265(15):1961-7.

7. Spohr HL, Willms J, Steinhausen HC. Fetal alcohol spectrum disorders in young adulthood. J Pediatr. 2007;150(2):175-9. 9 e1.

8. Chudley AE, Kilgour AR, Cranston M, Edwards M. Challenges of diagnosis in fetal alcohol syndrome and fetal alcohol spectrum disorder in the adult. Am J Med Genet C: Semin Med Genet. 2007;145C(3):261-72.

9. Temple VK, Ives J, Lindsay A. Diagnosing FASD in adults: the development and operation of an adult FASD clinic in Ontario, Canada. J Popul Ther Clin Pharmacol. 2015;22(1):e96-105.

10. Mattson SN, Crocker N, Nguyen TT. Fetal alcohol spectrum disorders: neuropsychological and behavioral features. Neuropsychol Rev. 2011;21(2):81-101.

11. Day NL, Helsel A, Sonon K, Goldschmidt L. The association between prenatal alcohol exposure and behavior at 22 years of age. Alcohol Clin Exp Res. 2013;37(7):1171-8.

12. Connor PD, Sampson PD, Streissguth AP, Bookstein FL, Barr HM. Effects of prenatal alcohol exposure on fine motor coordination and balance: a study of two adult samples. Neuropsychologia. 2006;44(5):744-51.

13. Connor PD, Streissguth AP, Sampson PD, Bookstein FL, Barr HM. Individual differences in auditory and visual attention among fetal 
alcohol-affected adults. Alcohol Clin Exp Res. 1999;23(8):1395402.

14. Coles CD, Lynch ME, Kable JA, Johnson KC, Goldstein FC. Verbal and nonverbal memory in adults prenatally exposed to alcohol. Alcohol Clin Exp Res. 2010;34(5):897-906.

15. Rangmar J, Sandberg AD, Aronson M, Fahlke C. Nord J Psychiatry. 2015:1-7. 2015.1009487. Adults with FAS have poorer short-term memory, working memory, planning ability, theory of mind, and a reduced sense of coherence compared to adult controls.

16. Kodituwakku PW, Handmaker NS, Cutler SK, Weathersby EK, Handmaker SD. Specific impairments in self-regulation in children exposed to alcohol prenatally. Alcohol Clin Exp Res. 1995;19(6): 1558-64.

17. Streissguth AP, Bookstein FL, Barr HM, Sampson PD, O'Malley K, Young JK. Risk factors for adverse life outcomes in fetal alcohol syndrome and fetal alcohol effects. J Dev Behav Pediatr. 2004;25(4):228-38.

18. Famy C, Streissguth AP, Unis AS. Mental illness in adults with fetal alcohol syndrome or fetal alcohol effects. Am J Psychiatry. 1998;155(4):552-4.

19. Barr HM, Bookstein FL, O'Malley KD, Connor PD, Huggins JE, Streissguth AP. Binge drinking during pregnancy as a predictor of psychiatric disorders on the Structured Clinical Interview for DSMIV in young adult offspring. Am J Psychiatry. 2006;163(6):1061-5.

20. Streissguth A, Barr H, Kogan J, Bookstein F. Understanding the occurrence of secondary disabilities in clients with fetal alcohol syndrome (FAS) and fetal alcohol effects (FAE). Final report to the Centers for Disease Control and Prevention (CDC). 1996;9606.

21. Clark E, Lutke J, Minnes P, Ouellette-Kuntz H. Secondary disabilities among adults with fetal alcohol spectrum disorder in British Columbia. J FAS Int. 2004;2(e13):1-12.

22. Temple V, Shewfelt L, Tao L, Casati J, Klevnick L, Place S. Comparing daily living skills in adults with fetal alcohol spectrum disorders (FASD) to and IQ matched clinical sample. J Popul Ther Clin Pharmacol. 2011;18(2):e397-402.

23. Rangmar J, Hjern A, Vinnerljung B, Stromland K, Aronson M, Fahlke C. Psychosocial outcomes of fetal alcohol syndrome in adulthood. Pediatrics. 2015;135(1):e52-8. Data from the National Registry in Sweden indicates that adults with FAS have more difficulties associated with secondary disorders than do adults in the general population.

24. Lebel C, Roussotte F, Sowell ER. Imaging the impact of prenatal alcohol exposure on the structure of the developing human brain. Neuropsychol Rev. 2011;21(2):102-18.

25. Chen X, Coles CD, Lynch ME, Hu X. Understanding specific effects of prenatal alcohol exposure on brain structure in young adults. Hum Brain Mapp. 2012;33(7):1663-76.

26. Wozniak JR, Muetzel RL. What does diffusion tensor imaging reveal about the brain and cognition in fetal alcohol spectrum disorders? Neuropsychol Rev. 2011;21(2):133-47.

27. Ma X, Coles CD, Lynch ME, LaConte SM, Zurkiya O, Wang D, et al. Evaluation of corpus callosum anisotropy in young adults with fetal alcohol syndrome according to diffusion tensor imaging. Alcohol Clin Exp Res. 2005;29(7):1214-22.

28. Li L, Coles CD, Lynch ME, Hu X. Voxelwise and skeleton-based region of interest analysis of fetal alcohol syndrome and fetal alcohol spectrum disorders in young adults. Hum Brain Mapp. 2009;30(10):3265-74.

29. Santhanam P, Li Z, Hu X, Lynch ME, Coles CD. Effects of prenatal alcohol exposure on brain activation during an arithmetic task: an fMRI study. Alcohol Clin Exp Res. 2009;33(11):1901-8.

30. Santhanam P, Coles CD, Li Z, Li L, Lynch ME, Hu X. Default mode network dysfunction in adults with prenatal alcohol exposure. Psychiatry Res. 2011;194(3):354-62.
31. Broyd SJ, Demanuele C, Debener S, Helps SK, James CJ, SonugaBarke EJ. Default-mode brain dysfunction in mental disorders: a systematic review. Neurosci Biobehav Rev. 2009;33(3):279-96.

32. Oleson DR, Magee RM, Donahoe RM, Falek A, Coles CD. Immunity and prenatal alcohol exposure. A pilot study in human adolescents. Adv Exp Med Biol. 1998;437:255-64.

33. Johnson S, Knight R, Marmer DJ, Steele RW. Immune deficiency in fetal alcohol syndrome. Pediatr Res. 1981;15(6):908-11.

34. Castells S, Mark E, Abaci F, Schwartz E. Growth retardation in fetal alcohol syndrome. Unresponsiveness to growth-promoting hormones. Dev Pharmacol Ther. 1981;3(4):232-41.

35. Burd L, Peterson L, Kobrinsky N. Fetal alcohol spectrum disorders and childhood cancer: a concise review of case reports and future research considerations. Pediatr Blood Cancer. 2014;61(5):768-70.

36. Barker DJ. Sir Richard Doll lecture. Developmental origins of chronic disease. Public Health. 2012;126(3):185-9.

37. McGill J, Meyerholz DK, Edsen-Moore M, Young B, Coleman RA, Schlueter AJ, et al. Fetal exposure to ethanol has long-term effects on the severity of influenza virus infections. J Immunol. 2009;182(12):7803-8.

38. Zhang X, Lan N, Bach P, Nordstokke D, Yu W, Ellis L, et al. Prenatal alcohol exposure alters the course and severity of adjuvant-induced arthritis in female rats. Brain Behav Immun. 2012;26(3):439-50.

39. Chen L, Nyomba BL. Effects of prenatal alcohol exposure on glucose tolerance in the rat offspring. Metab Clin Exp. 2003;52(4): 454-62.

40. Pennington JS, Shuvaeva TI, Pennington SN. Maternal dietary ethanol consumption is associated with hypertriglyceridemia in adult rat offspring. Alcohol Clin Exp Res. 2002;26(6):848-55.

41. Nordestgaard BG, Varbo A. Triglycerides and cardiovascular disease. Lancet. 2014;384(9943):626-35. doi:10.1016/s01406736(14)61177-6.

42. Polanco TA, Crismale-Gann C, Reuhl KR, Sarkar DK, Cohick WS Fetal alcohol exposure increases mammary tumor susceptibility and alters tumor phenotype in rats. Alcohol Clin Exp Res. 2010;34(11): 1879-87.

43. Murugan S, Zhang C, Mojtahedzadeh S, Sarkar DK. Alcohol exposure in utero increases susceptibility to prostate tumorigenesis in rat offspring. Alcohol Clin Exp Res. 2013;37(11):1901-9.

44. Catts VS, Fung SJ, Long LE, Joshi D, Vercammen A, Allen KM, et al. Rethinking schizophrenia in the context of normal neurodevelopment. Front Cell Neurosci. 2013;7:60.

45. Lebel C, Gee M, Camicioli R, Wieler M, Martin W, Beaulieu C. Diffusion tensor imaging of white matter tract evolution over the lifespan. NeuroImage. 2012;60(1):340-52.

46. Lebel C, Mattson SN, Riley EP, Jones KL, Adnams CM, May PA, et al. A longitudinal study of the long-term consequences of drinking during pregnancy: heavy in utero alcohol exposure disrupts the normal processes of brain development. J Neurosci. 2012;32(44): $15243-51$

47. Treit S, Lebel C, Baugh L, Rasmussen C, Andrew G, Beaulieu C. Longitudinal MRI reveals altered trajectory of brain development during childhood and adolescence in fetal alcohol spectrum disorders. J Neurosci. 2013;33(24):10098-109.

48. Gautam P, Nunez SC, Narr KL, Kan EC, Sowell ER. Effects of prenatal alcohol exposure on the development of white matter volume and change in executive function. NeuroImage Clin. 2014;5: 19-27.

49. Giedd JN, Raznahan A, Alexander-Bloch A, Schmitt E, Gogtay N, Rapoport JL. Child psychiatry branch of the National Institute of Mental Health longitudinal structural magnetic resonance imaging study of human brain development. Neuropsychopharmacol. 2015;40(1):43-9.

50. Shaw P, Eckstrand K, Sharp W, Blumenthal J, Lerch JP, Greenstein $\mathrm{D}$, et al. Attention-deficit/hyperactivity disorder is characterized by 
a delay in cortical maturation. Proc Natl Acad Sci U S A. 2007;104(49):19649-54.

51. Barbaresi WJ, Colligan RC, Weaver AL, Voigt RG, Killian JM, Katusic SK. Mortality, ADHD, and psychosocial adversity in adults with childhood ADHD: a prospective study. Pediatrics. 2013;131(4):637-44.

52. Hart H, Rubia K. Neuroimaging of child abuse: a critical review. Front Hum Neurosci. 2012;6:52.

53. Dykens EM. Aging in rare intellectual disability syndromes. Dev Disabil Res Rev. 2013;18(1):75-83.

54. Zigman WB. Atypical aging in Down syndrome. Dev Disabil Res Rev. 2013;18(1):51-67.

55. Ness KK, Krull KR, Jones KE, Mulrooney DA, Armstrong GT, Green DM, et al. Physiologic frailty as a sign of accelerated aging among adult survivors of childhood cancer: a report from the St Jude Lifetime cohort study. J Clin Oncol Off J Am Soc Clin Oncol. 2013;31(36):4496-503.

56. Tyrka AR, Price LH, Kao HT, Porton B, Marsella SA, Carpenter LL. Childhood maltreatment and telomere shortening: preliminary support for an effect of early stress on cellular aging. Biol Psychiatry. 2010;67(6):531-4.

57. Moretti L, Cristofori I, Weaver SM, Chau A, Portelli JN, Grafman J. Cognitive decline in older adults with a history of traumatic brain injury. Lancet Neurol. 2012;11(12):1103-12.

58. Tucker AM, Stern Y. Cognitive reserve in aging. Curr Alzheimer Res. 2011;8(4):354-60.

59. Stern Y. Cognitive reserve in ageing and Alzheimer's disease. The Lancet Neurol. 2012;11(11):1006-12. 